\title{
Efecto de una intervención educativa en la calidad de vida del paciente hipertenso
}

\author{
A dalberto Jorge Barrón-Rivera, M.C., ${ }^{(1)}$ Federico Luis Torreblanca-Roldán, M.C., ${ }^{(2)}$ \\ Landy Isabel Sánchez-Casanova, M.C., ${ }^{(2)}$ Miguel Martínez-Beltrán, M.C.(2)
}

\begin{abstract}
Barrón-Rivera AJ, Torreblanca-Roldán FL, Sánchez-Casanova LI, Martínez-Beltrán M. Efecto de una intervención educativa en la calidad de vida del paciente hipertenso. Salud Publica Mex 1998;40:503-509.
\end{abstract}

\section{Resumen}

Objetivo. Evaluar el efecto de una intervención educativa sobre la calidad de vida del paciente hipertenso. Material y métodos. Se estudiaron 150 adultos hipertensos en un primer nivel de atención mediante un ensayo clínico aleatorizado a dos grupos. El grupo experimental fue objeto de una inter vención educativa so bre hipertensión y la repercusión del estilo de vida en el control de tal enfermedad. El grupo testigo no recibió intervención. En ambos grupos se determinó la calidad de vida mediante una escala análoga visual, antes y seis meses después de la intervención educativa. Los datos se analizaron mediante las pruebas t-pareada yW ilcoxon. Resultados. El grado de mejoría, en relación con la fuerza física y el estado anímico, difirió entre grupos $(p<0.05)$; en las áreas correspondientes a la capacidad de pensamiento, la convivencia social-familiar, la percepción de calidad de vida y el funcionamiento sexual, sólo el grupo experimental mostró cambios $(p<0.05)$. Conclusiones. La inter vención educativa es efectiva para modificar la calidad de vida del paciente hipertenso.

Palabras clave: hipertensión; intervención educativa; calidad de vida; México
Barrón-Rivera AJ, Torreblanca-Roldán FL, Sánchez-Casanova LI, Martínez-Beltrán M. Effect of an educational intervention on the quality of life of the hypertense patient. Salud Publica Mex 1998;40:503-509.

\begin{abstract}
A bstract
Objective. To evaluate the effect of an educational program on the quality of life of the hypertense patient. Material and methods. A randomized clinical trial was performed on 150 adult hypertense patients who were divided into 2 groups.T he experimental group received a short educational program on hypertension and the effects on the patients life style, specifically concerning their control of the disease. The control group did not receive the educational program. Q uality of life of both groups was determined by an analogous visual scale, before and 6 months after the educational program. Data were analyzed by the paired t- and W ilcoxon tests. Results. The degree of improvement with respect to physical strength and emotional condition differed between groups $(p<0.05)$. In the areas of thought capacity, social participation, perceived quality of life and sexual function only the experimental group showed changes $(p<0.05)$. Conclusions. An educational program is effective to modify the quality of life of the hypertense patient.
\end{abstract}

Key words: hypertension; educational program; quality of life; Mexico

\begin{abstract}
a hipertensión arterial es una patología crónica degenerativa presente en $20 \%$ de las personas de entre 18 y 65 años de edad del mundo. Es un factor de riesgo importante para las enfermedades cardiovasculares, cerebrovasculares, renales y vasculares periféricas. ${ }^{1}$
\end{abstract}

El manejo no farmacológico, es decir, la modificación del estilo de vida está indicada como medida inicial, o bien, como suplementaria al manejo con medicamentos para el paciente con hipertensión arterial; incluye la disminución en la ingesta de sodio, alcohol y grasas saturadas, así como el incremento de la acti-

(1) Unidad de Medicina Familiar $N^{\circ} 56$, Instituto Mexicano del Seguro Social (IMSS), Mérida, Yucatán, México.

(2) Unidad de Medicina Familiar $N^{\circ} 13$, IMSS, Mérida, Yucatán, México.

Fecha de recibido: 9 de enero de 1998 - Fecha de aprobado: 10 de julio de 1998

Solicitud de sobretiros: Dr. Adalberto Jorge Barrón Rivera. Avenida Alemán 108 X 36A, colonia Jesús Carranza, 97140 Mérida, Yucatán, México. 
vidad física durante el tiempo libre del hipertenso., ${ }^{2,3}$ Este manejo es efectivo para disminuir las cifras de presión arterial de las personas con niveles normales en límites altos ${ }^{4} \mathrm{y}$ en los pacientes con hipertensión. ${ }^{5-8}$

Al evaluar el impacto de la enfermedad y del tratamiento, es esencial medir y evaluar el grado de alivio de los síntomas, mismos que podrían traducirse en una mejoría de la calidad de vida $(\mathrm{CV}){ }^{9}$ considerada esta última como la sensación de bienestar físico y de satisfacción emocional, psíquica y social del individuo. ${ }^{10}$ Para evaluar la CV se emplean instrumentos que abarcan diferentes dominios relacionados con los valores subjetivos del paciente, determinados a su vez por los contextos sociales, culturales y económicos, que también se ven afectados por la enfermedad o por el tratamiento mismo. La respuesta al tratamiento, su duración, la severidad de la enfermedad y el estado y la magnitud de los cambios esperados son criterios que influyen en la elección de los componentes de la CV que se van a evaluar. El uso de escalas visuales análogas implica que la intensidad o la frecuencia de un síntoma puede representarse con una escala linear continua, y que el respondente de la escala también entiende esta representación. ${ }^{11}$

Aunque la hipertensión arterial leve a moderada tiene pocas manifestaciones sintomáticas por sí misma, los efectos secundarios del manejo están asociados con la poca adherencia o el franco abandono del tratamiento medicamentoso. Para algunos pacientes el hecho de ingerir medicamento implica un problema más grande que la enfermedad misma; a pesar de la frecuencia con que se presenta esta situación, pocos estudios clínicos han intentado evaluar los diferentes aspectos de la CV en este tipo de pacientes. ${ }^{12}$

En este estudio, mediante un ensayo clínico aleatorizado, se evaluó el efecto de una intervención educativa sobre la calidad de vida del paciente con hipertensión arterial leve a moderada.

\section{Material y métodos}

Del 4 noviembre de 1996 al 31 julio de 1997, se realizó un ensayo clínico aleatorizado en pacientes hipertensos de la Unidad de Medicina Familiar No. 13 Chuburná, del Instituto Mexicano del Seguro Social (IMSS).

Los pacientes fueron seleccionados mediante el método no probabilístico por conveniencia, posteriormente a su envío al Módulo del Hipertenso. En el estudio se incluyeron pacientes de ambos sexos, en edades de entre 25 y 70 años, portadores de hipertensión arterial esencial leve y moderada -de acuerdo con lo establecido por el Joint National Committe on Detection, Evaluation and Treatment of High Blood Pressure-, ${ }^{2}$ que estuviesen o no bajo tratamiento con medicamentos para controlar la presión arterial y que pudieran hacer modificaciones del estilo de vida (MEV). No se incluyeron los pacientes con historia o evidencia de lesión severa en algún órgano blanco (enfermedad arterial coronaria, disfunción ventricular izquierda o insuficiencia cardia$\mathrm{ca}$, isquemia cerebral transitoria o apoplejía, ausencia de pulsos en las extremidades con o sin claudicación intermitente, e insuficiencia renal), los portadores de hipertensión arterial secundaria, las mujeres embarazadas, los diabéticos, los que rechazaron el manejo de MEV, los que tuvieran algún impedimento físico o mental para efectuar las MEV y los obesos severos (índice de masa corporal superior a $40 \mathrm{~kg} / \mathrm{m}^{2}$ ).

El tamaño de la muestra se calculó mediante la fórmula para comparar proporciones, ${ }^{13}$ con un nivel de significancia de 0.05 y con un poder de 0.80 , para detectar una diferencia de $22 \%$ en el cambio de la calidad de vida entre los grupos después del tratamiento, de tal modo que se obtuvo un total de 65 pacientes por grupo de tratamiento.

Se hicieron las mediciones y el examen clínico iniciales en los pacientes que reunieron los criterios de selección, después de haber expuesto los objetivos del estudio y obtenido la aceptación y la firma de consentimiento informado. La presión arterial se midió en las visitas inicial y subsecuentes después de que el paciente estuvo sentado y en reposo por cinco minutos y siempre en su brazo derecho. Se efectuaron dos mediciones con diferencia de dos minutos entre cada una, con un esfigmomanómetro mercurial marca Adex, calibrado a cero $\mathrm{mm} \mathrm{Hg}$. Se registró el promedio de las dos lecturas como medida de presión arterial, usando como referencia el primero y quinto ruidos de Korotkoff para la lectura sistólica y diastólica, respectivamente.

El peso se determinó a partir del medio kilo más cercano, en una báscula clínica calibrada marca Bame, y con el paciente descalzo y con ropa mínima. La estatura se midió igualmente tomando como base el medio centímetro más cercano, utilizando el estadímetro de la báscula clínica y con el paciente en estiramiento máximo y descalzo. El tiempo de actividad física durante el tiempo libre se determinó mediante el interrogatorio al paciente sobre el promedio en minutos de la actividad física desarrollada en la semana previa a cada cita, para tratar de evitar el sesgo de memoria.

Los pacientes fueron asignados a dos grupos usando una lista de números aleatorios obtenida de la computadora mediante el paquete estadístico True Epistat, versión 1987; un grupo siguió con el manejo antihipertensivo establecido por su médico familiar y, al otro, además del manejo medicamentoso de base, se le aplicó la intervención educativa. 
Esta última consistió en seis sesiones individuales mensuales en las que se proporcionó al paciente información completa y orientada a disminuir $250 \mathrm{kcal}$ del aporte calórico por día de acuerdo con los requerimientos correspondientes, según peso, talla, sexo y edad. ${ }^{14}$ Aunado a ello, se propició la disminución de la ingesta de productos alimenticios con alto contenido de sal y grasas saturadas de origen animal, además de que se brindó orientación y asesoría para desarrollar, durante el tiempo libre, actividad física de tipo isométrico como la marcha; se sugirió efectuarla durante 150 minutos en promedio por semana, divididos en cinco sesiones de 30 minutos como mínimo y con una intensidad regulada por la sensación subjetiva de fatiga.

En la primera sesión, y para reforzar las indicaciones, a cada participante se le proporcionó un folleto con información sobre la hipertensión, su etiología, su manejo medicamentoso y no medicamentoso, y sus complicaciones, así como sobre algunos aspectos del ambiente susceptibles de modificación por parte del paciente (consumir dieta alta en fibra, disminuir la ingestión de sal y de grasas saturadas de origen animal, controlar el peso e incrementar la actividad física). El folleto incluía también cuadros del contenido calórico de los alimentos de mayor consumo en la región.

Ambos grupos de pacientes fueron citados cada mes para valorar sus condiciones clínicas y hacer el seguimiento del manejo, de la prescripción medicamentosa, de las mediciones de peso y de la presión arterial, así como para hacer un interrogatorio sobre tiempo de actividad física desarrollada. El grupo control hizo su seguimiento con su médico familiar, y el grupo de intervención, en el Módulo del Hipertenso, coordinado por un investigador del estudio.

Para mantener una tasa de seguimiento aceptable del plan de manejo, se establecieron estrategias de educación para el paciente respecto a las expectativas que podía tener sobre su enfermedad, se fomentó la retroinformación sobre las variaciones que presentaron las cifras de presión arterial y, junto con el paciente, se establecieron las metas del manejo y la individualización del tratamiento.

El efecto de la intervención educativa se evaluó a partir de la modificación de las cifras de presión arterial, del peso y del tiempo destinado a la actividad física.

La CV se evaluó mediante la escala análoga visual "indice de cambio en la calidad de vida", ${ }^{15}$ un instrumento fácil de entender para el paciente y con un tiempo de aplicación breve; el instrumento original consta de siete reactivos que exploran las áreas física, mental, de actividad cotidiana y emocional y de convivencia. Además, se complementó con el reactivo “En relación con su funcionamiento sexual, ahora se siente usted...", destinado a evaluar la repercusión que tienen los medicamentos y la dieta en la vida sexual del paciente con hipertensión; 9,16 éste fue calificado igual que los reactivos originales, sobre una línea de $10 \mathrm{~cm}, \mathrm{y}$ con respuestas extremas de mucho peor a mucho mejor. Los instrumentos de la entrevista inicial y la final recibieron calificaciones de 0 a 10 por cada ítem, donde 0 correspondió a un máximo compromiso por el cuidado de la enfermedad, y 10, a un nulo compromiso; en la entrevista final se evaluó el cambio operado durante la intervención educativa. Para la aplicación del instrumento de medición fue necesario capacitar a una enfermera sanitarista, la cual desconocía el grupo de pertenencia de cada paciente. La medición se realizó en todos los pacientes, tanto antes de ser aleatorizados a los grupos, como al final del estudio.

\section{A nálisis estadístico}

Se empleó el análisis multivariado para comparar, entre grupos, cambios en la presión arterial, el peso y la actividad física desarrollada durante el tiempo libre.

Para evaluar la sensibilidad al cambio de la calidad de vida se aplicó la prueba paramétrica $t$-pareada complementada con la no paramétrica suma de rangos de Wilcoxon, de tal modo que se pudiera dar un puntaje global por paciente de acuerdo con sus rangos de respuesta. La evaluación de las diferencias de puntuación de calidad de vida entre grupos se analizó mediante la prueba de $t$ en muestras independientes, mientras que para identificar pacientes con cambio clínico objetivo después de la intervención se utilizó la fórmula:

$$
\mathrm{PC}=\frac{\mathrm{P}_{2}-\mathrm{P}_{1}}{\mathrm{P}_{1}} \text { donde }
$$

$\mathrm{PC}=$ porcentaje de cambio

$\mathrm{P}_{1}=$ puntaje de calidad de vida al inicio del estudio

$\mathrm{P}_{2}=$ puntaje de calidad de vida al final del estudio ${ }^{17}$

Para evaluar las diferencias del porcentaje de cambio entre los grupos se aplicó la prueba de $\chi^{2}$. Se efectuó un análisis estratificado de varianza de un factor para evaluar la influencia sobre la calidad de vida por tipo de medicamento (diurético, betabloqueador, inhibidor de la enzima convertidora de angiotensina, calcioantagonistas y agonistas centrales), tipo de actividad (hogar y pensionado, entre otras), estado civil (casado, viudo, etc.) y según la diferencia respecto a la medición inicial, en tiempo promedio, de la actividad física por semana (<30 min, 30 a $150 \mathrm{~min}$ y $>150 \mathrm{~min}$ por semana). 
Como criterio de significancia estadística se utilizó una probabilidad de 0.05 para pruebas no direccionales. Para el análisis descriptivo e inferencial de los datos se empleó el paquete estadístico SPSS para Windows, versión 5.0.1, 1992.

\section{Resultados}

En el cuadro I se resumen las características de los participantes aleatorizados a dos grupos. El promedio de edad de los 150 participantes fue de 51.8 \pm 9.6 años; 57 $(38 \%)$ pertenecían al sexo masculino y $93(62 \%)$, al femenino, con una escolaridad promedio de $6.7 \pm 4$ años, estatura de $1.54 \pm 0.1 \mathrm{~m}$, y un índice de masa corporal de $30.06 \pm 3.8 \mathrm{~kg} / \mathrm{m}^{2}$; la presión arterial fue de $141 \pm 17.5$ y $88.5 \pm 10.9 \mathrm{~mm} \mathrm{Hg}$ sistólica y diastólica, respectivamente; tuvieron un peso de $73.2 \pm 11.9 \mathrm{~kg}$ y un tiempo de actividad física en tiempo libre de $149 \pm 84.1 \mathrm{~min}$ por semana. Respecto al estado civil, 125 (83.3\%) eran casados; $18(12 \%)$, viudos; tres $(2 \%)$, solteros, y uno $(0.66 \%)$, divorciado. Entre las actividades principales, $68(45.3 \%)$ se dedicaban a las labores domésticas; 21 (14\%) eran pensionados; $13(8.6 \%)$, comerciantes, y $11(7.3 \%)$, obreros. Los grupos difirieron significativamente sólo en el tiempo de actividad física por semana $(p<0.001)$.

De 139 sujetos que terminaron el estudio, 20 (14.4\%) en ambos grupos tomaban calcioantagonistas (16, nifedipina, y cuatro, verapamilo); seis (4.3\%), agonistas centrales (alfametildopa); 30 (21.6\%), betabloqueadores (19, metoprolol, y 11, propranolol); $78(56.1 \%)$, inhibidores de la enzima convertidora de angiotensina (43, captopril, y 35, enalapril), y solamente cinco (3.6\%), diurético (clortalidona), sin que se hayan encontrado diferencias estadísticamente significativas por el tipo de medicamento entre los grupos.

En el grupo de intervención las citas se cumplieron en $96 \%(72 / 75)$ de los casos al primer mes, y en $90.66 \%$ (68/75), entre el segundo y el sexto mes de seguimiento. En el grupo control $98.6 \%$ (74/75) acudieron a sus citas durante el primer mes, $97.3 \%$ (73/75) en el segundo y $94.66 \%$ (71/75) entre el tercero y el sexto mes.

Al término del estudio, en el grupo experimental la presión arterial sistólica y diastólica, respectivamente, disminuyó 9.96/5.57, y en el control, $3 / 2.5 \mathrm{~mm}$ $\mathrm{Hg}$. La pérdida de peso en el grupo experimental fue de $0.74 \mathrm{~kg}$, mientras que en el control se incrementó en $0.4 \mathrm{~kg}$ en promedio. La duración de la actividad física aumentó a la semana 59 min en el experimental y 2.2 min en el control. Como cifra final se tomó el promedio de las últimas cinco lecturas para cada una de las variables en comparación; las diferencias entre los grupos en las variables anteriores analizadas mediante

\section{Cuadro I \\ Datos demográficos Y VARIABLES Clínicas EN LOS GRUPOS DEL ESTUDIO SOBRE CALIDAD DE VIDA DEL HIPERTENSO. MÉXICO, 1996-1997}

\begin{tabular}{|c|c|c|}
\hline Grupos & $\begin{array}{c}\text { Intervención } \\
\quad(n=75)\end{array}$ & $\begin{array}{l}\text { Control } \\
(n=75)\end{array}$ \\
\hline Sexo masculino, $\mathrm{n}(\%)$ & $30(40)$ & $27(36)$ \\
\hline Edad, años \pm DE & $52.2 \pm 9.83$ & $51.4 \pm 9.39$ \\
\hline Escolaridad, años $\pm D E$ & $6.36 \pm 4.35$ & $7.09 \pm 3.68$ \\
\hline O cupación: Hogar, n (\%) & $40(53.33)$ & $28(37.3)$ \\
\hline Pensionado, $\mathrm{n}(\%)$ & $8(10)$ & $13(17.3)$ \\
\hline Estado civil: C asado, n (\%) & $61(81.3)$ & $64(85.3)$ \\
\hline Viudo, n (\%) & $11(14.6)$ & $7(9.33)$ \\
\hline Presión arterial sistólica, $\mathrm{mm}$ de $\mathrm{Hg} \pm \mathrm{DE}$ & $141 \pm 14.6$ & $142 \pm 20$ \\
\hline Presión arterial diastólica, mm de $\mathrm{Hg} \pm \mathrm{DE}$ & $87.5 \pm 11$ & $89.5 \pm 10.8$ \\
\hline Peso, $\mathrm{kg} \pm \mathrm{DE}$ & $72 \pm 12.1$ & $74.4 \pm 11.6$ \\
\hline $\begin{array}{l}\text { Actividad física en tiempo libre, } \\
\text { min por semana } \pm D E *\end{array}$ & $175 \pm 107$ & $124 \pm 37$ \\
\hline \multicolumn{3}{|c|}{$\begin{array}{l}* p=0.0001 \text {, basado en la prueba de t para comparar medias de grupos } \\
\text { independientes } \\
D E \text { : desviación estándar }\end{array}$} \\
\hline
\end{tabular}

la $t$-pareada fueron estadísticamente significativas a favor del grupo experimental $(p<0.05)$. Al incluir todas las mediciones realizadas durante el seguimiento se encontró, mediante el análisis multivariado, que las diferencias por grupo fueron estadísticamente significativas para la presión arterial diastólica y sistólica, el peso y el tiempo de actividad física (todas con $p<0.01$ ). No se encontraron diferencias por tipo de medicamento, estado civil, ocupación o tiempo de actividad física.

En el cuadro II se muestran los cambios ocurridos en los puntajes sobre calidad de vida en ambos grupos, según el área explorada, antes y después de la intervención educativa. Respecto a la fuerza física y al estado anímico, ambos grupos tuvieron diferencias estadísticamente significativas en comparación con la puntuación basal; en las áreas de capacidad de pensamiento, convivencia social-familiar, percepción de la calidad de vida y funcionamiento sexual, únicamente el grupo experimental mostró cambios, mismos que fueron estadísticamente significativos $(p<0.05)$. En el área de ayuda para actividades diarias y sintomatología hipertensiva no se encontraron diferencias en ambos grupos. El grado de mejoría difirió entre grupos significativamente en lo tocante a la fuerza física $(p=0.04)$ y al estado anímico $(p=0.03)$; para el resto de las áreas exploradas, el grado de mejoría no mostró cambios significativos después de la intervención educativa. 
Cuadro II

Puntuación promedio por grupos de los participantes en el estudio sobre LA CALIDAd DE VIDA DEL HIPERTENSO. MéXICO, 1996-1997*

\begin{tabular}{|c|c|c|c|c|c|c|}
\hline Reactivo & Grupo & Antes (DE) & Después (DE) & Cambio (DE) & $p^{\ddagger}$ & $p^{\S}$ \\
\hline \multirow[t]{2}{*}{ 1. Fuerza física } & Experimental & $8.22(1.39)$ & $8.58(1.22)$ & $0.36(1.02)$ & 0.004 & 0.0086 \\
\hline & Control & $8.30(1.22)$ & $8.40(1.12)$ & $0.09(0.45)$ & 0.070 & 0.074 \\
\hline \multirow[t]{2}{*}{ 2. Capacidad de pensamiento } & Experimental & $8.53(1.2)$ & $8.70(1.08)$ & $0.17(0.66)$ & 0.033 & 0.033 \\
\hline & Control & $8.12(1.30)$ & $8.14(1.26)$ & $0.01(0.31)$ & NS & NS \\
\hline \multirow[t]{2}{*}{ 3. Ayuda para actividades } & Experimental & $9.37(1.0)$ & $9.44(0.95)$ & $0.07(0.39)$ & NS & NS \\
\hline & Control & $8.49(1.6)$ & $8.54(1.62)$ & $0.05(0.37)$ & NS & NS \\
\hline \multirow[t]{2}{*}{ 4. Estado anímico } & Experimental & $7.68(2.03)$ & $8.11(1.89)$ & $0.42(1.02)$ & 0.001 & 0.005 \\
\hline & Control & $7.87(1.39)$ & $8.01(1.25)$ & $0.14(0.48)$ & 0.017 & NS \\
\hline \multirow[t]{2}{*}{ 5. Convivencia social-familiar } & Experimental & $8.33(1.95)$ & $8.58(1.55)$ & $0.25(0.92)$ & 0.028 & 0.039 \\
\hline & Control & $8.19(1.57)$ & $8.25(1.49)$ & $0.05(0.41)$ & NS & NS \\
\hline \multirow[t]{2}{*}{ 6. Sintomatología hipertensiva } & Experimental & $7.89(1.81)$ & $8.20(1.77)$ & $0.22(1.06)$ & NS & NS \\
\hline & Control & $7.74(1.52)$ & $7.81(1.42)$ & $0.07(0.78)$ & NS & NS \\
\hline \multirow[t]{2}{*}{ 7. Percepción de calidad de vida } & Experimental & $7.85(1.87)$ & $8.05(1.70)$ & $0.20(0.83)$ & 0.046 & 0.05 \\
\hline & Control & $7.84(1.37)$ & $8.01(1.29)$ & $0.07(0.39)$ & NS & NS \\
\hline \multirow[t]{2}{*}{ 8. Funcionamiento sexual } & Experimental & $7.92(2.17)$ & $8.10(2.09)$ & $0.17(0.57)$ & 0.013 & 0.016 \\
\hline & Control & $7.46(2.11)$ & $7.56(2.10)$ & $0.09(0.51)$ & NS & NS \\
\hline
\end{tabular}

\footnotetext{
* Se incluyeron 68 participantes del grupo experimental y 71 del grupo control que terminaron el estudio a los seis meses.

₹ Prueba de t-pareada con alfa de 0.05 .

$\S$ Prueba de rangos señalados y pares igualados de W ilcoxon con alfa de 0.05 .

DE: desviación estándar

N S: no significativo.
}

Los cambios en la calidad de vida analizados mediante el análisis estratificado de varianza de un factor fueron estadísticamente significativos sólo para el tiempo de actividad física, que pasó de 30 a 150 $\min (p=0.046)$ y de 151 a 350 min en promedio por semana $(p=0.018)$, sin que se encontraran diferencias significativas para la ocupación, el estado civil y el tipo de medicamento antihipertensivo empleado.

Veintinueve pacientes $(42.6 \%)$ del grupo experimental y $38(53 \%)$ del grupo control informaron efectos secundarios leves (no interfieren con las actividades diarias del individuo) atribuibles al medicamento. La fatiga, la ansiedad, la tos, la cefalea, la náusea y el mareo fueron los efectos secundarios más frecuentemente notificados por los pacientes, aunque ninguno fue reportado por más del $14 \%$ de ellos (cuadro III).

En el cuadro IV se muestra el número de pacientes con cambio clínico objetivo por área de evaluación; en el grupo experimental se observó un número mayor de pacientes con cambio clínico objetivo, y únicamente el área de estado anímico mostró una diferencia significativa del cambio entre los grupos $(p<0.001)$.

\section{Discusión}

Para el clínico uno de los múltiples objetivos al evaluar la calidad de vida es establecer el bienestar subjetivo de los pacientes, su capacidad de realizar actividades sociales y su capacidad funcional después de recibir una intervención terapéutica, determinando si esta última tiene buena aceptación entre los pacientes que la reciben, sobre todo cuando estos resultados son comparados con un grupo de referencia.

Los datos globales al final del presente estudio demuestran una sensibilidad al cambio en seis de las ocho áreas exploradas, que son: el estado de ánimo, la fuerza física, la capacidad de pensamiento, la convivencia social-familiar, el funcionamiento sexual y la percepción de mejoría en la calidad de vida. Las áreas que no se modificaron fueron la sintomatología hipertensiva y la de ayuda para actividades diarias. En cuanto a los datos específicos por área de exploración, se encontró que el cambio clínico objetivo en el estado anímico fue más evidente y abarcó un mayor número de pacientes del grupo experimental, con una diferencia estadísticamente significativa en comparación con el grupo 


\section{Cuadro III \\ Principales efectos secundarios reportados POR LOS PARTICIPANTES DURANTE EL ESTUDIO SOBRE CALIDAD DE VIDA DEL HIPERTENSO. MÉxıco, 1996-1997*}

\begin{tabular}{|c|c|c|c|c|}
\hline \multirow[t]{2}{*}{ Efecto secundario } & \multicolumn{2}{|c|}{$\begin{array}{l}\text { Grupo experimental } \\
\quad(n=68)\end{array}$} & \multicolumn{2}{|c|}{$\begin{array}{c}\text { Grupo control } \\
\quad(n=71)\end{array}$} \\
\hline & Antes & Después & Antes & Después \\
\hline C efalea & 6 & 0 & 3 & 1 \\
\hline Estreñimiento & 4 & 3 & 3 & 1 \\
\hline Disgeusia & 4 & 0 & 4 & 3 \\
\hline Fatiga & 9 & 4 & 8 & 7 \\
\hline Insomnio & 3 & 0 & 4 & 1 \\
\hline Mareo & 6 & 0 & 4 & 1 \\
\hline Náusea & 4 & 0 & 6 & 3 \\
\hline Ansiedad & 10 & 3 & 6 & 4 \\
\hline Palpitaciones & 4 & 0 & 3 & 1 \\
\hline Tos & 13 & 6 & 7 & 7 \\
\hline Problemática sexual $\left.\right|^{\ddagger}$ & 7 & 6 & 4 & 3 \\
\hline
\end{tabular}

Cuadro IV

Número de pacientes con Cambio clínico objetivo AL FINAL DEL ESTUDIO SOBRE CALIDAD DE VIDA Del hIPERTenso. MÉxico, 1996-1997*

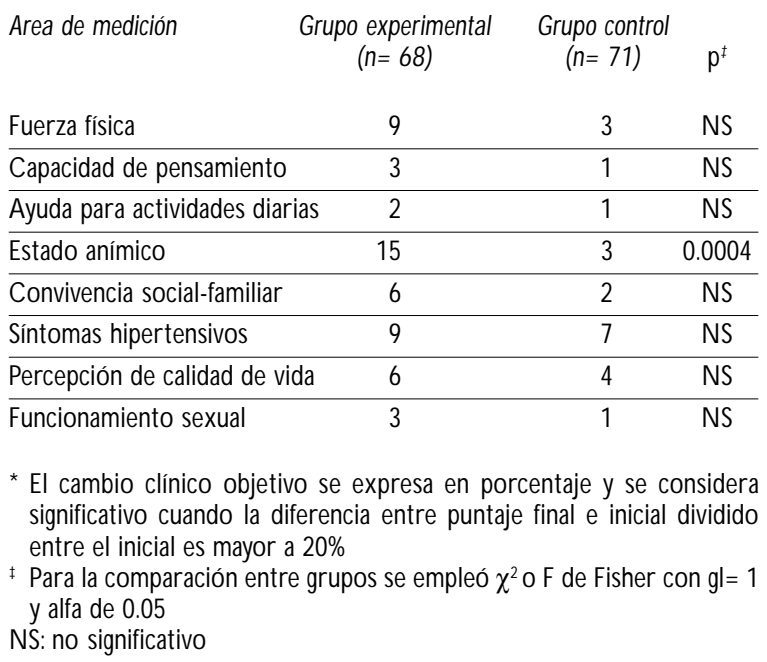

control $(p<0.001)$. Dichos cambios fueron más significativos en áreas como la convivencia social, la capacidad física y el estado de ánimo en los pacientes que efectuaron un promedio de 30 a 150 min más de actividad física por semana $(p<0.05)$, independientemente del grupo de estudio.

En este estudio la sintomatología referida por ambos grupos de pacientes se atribuyó sobre todo a efectos secundarios por el uso de medicamentos, y no mostró diferencias significativas de cambio por grupo al término del estudio.

Si bien no formaba parte de los propósitos de este estudio, se encontró que $25(58 \%)$ de los pacientes bajo manejo con captopril, 25 (71.4\%) con enalapril, $10(52.6 \%)$ con metoprolol, dos (40\%) con clortalidona y cinco $(31.5 \%)$ con nifedipina, presentaban efectos secundarios.

En este estudio, los pacientes que tomaban captopril y propranolol mostraron las mayores puntuaciones para la calidad de vida, a diferencia de lo indicado en el estudio TOMHS, ${ }^{6,18-19}$ donde los pacientes que tomaban clortalidona y acebutolol fueron los que más mejoraron su calidad de vida. En el estudio de Kostis ${ }^{7}$ los pacientes que recibieron un manejo no farmacológico y propranolol tuvieron una mejoría de la calidad de vida contra los que recibieron placebo en un estudio durante 12 semanas. Asimismo, en el estudio de Croog, ${ }^{9}$ cuya duración fue la misma que la de este estudio, los cambios fueron también a favor del captopril, seguidos por el propranolol, contra los encontrados con la metildopa.

Dentro de las limitantes del estudio, se considera que el diseño metodológico abierto pudo influir en el comportamiento de los pacientes al conocer el grupo de pertenencia, favoreciendo la aparición del efecto Haw thorne e influyendo en los resultados observados. El ingreso mensual promedio per cápita de los pacientes (\$398.00), tanto del grupo control como del grupo de intervención, fue otra limitante que pudo actuar, por un lado, con una pobre respuesta a la modificación dietética y, por consiguiente, con una mínima disminución ponderal, y por el otro, con una escasa asistencia a las reuniones-discusiones grupales, planteadas originalmente para desarrollarse durante el primer mes del estudio, debido al costo del transporte urbano, lo que llevó a modificar la estrategia educativa, conservando únicamente las sesiones mensuales individuales.

Cabe mencionar que en los estudios anteriores, a diferencia del presente, se emplearon cuestionarios estructurados para medir las diferentes áreas de la calidad de vida, los cuales incluían un alto número de ítems para evaluar cada área o dominio en particular, mientras que en este estudio se empleó una escala visual análoga con un número de ítems equivalente al número de dominios explorados y más acorde con 
el tipo de pacientes y los tiempos que se manejan en el ámbito institucional local; además, se considera que dicho instrumento puede retroalimentar al clínico de una manera rápida y confiable acerca de la repercusión del tratamiento en el paciente.

Los hallazgos de esta investigación sugieren que este tipo de estrategia educativa aplicada al paciente con una enfermedad crónico-degenerativa como la hipertensión, ayuda a mejorar el entendimiento de las terapias específicas y las metas de tratamiento, pues se corrigen conceptos erróneos, se incrementa la adherencia terapéutica, se favorece el bienestar y se provoca la sensación de una mejor calidad de vida del paciente. Este tipo de estrategia puede ser proporcionada por el equipo multidisciplinario de salud (médico familiar, trabajo social, medicina preventiva, dietología) en el primer nivel de atención médica por medio del Club del Hipertenso, donde se puede aplicar un tipo de enseñanza activo y participativo con la intervención del paciente en la elección del manejo y la determinación de metas individuales de manejo. ${ }^{20}$

A pesar de las limitaciones que el instrumento utilizado pueda tener para explorar la calidad de vida, al comparar los resultados del estudio con los de la literatura de referencia, se considera que una intervención educativa es efectiva para modificar la calidad de vida del hipertenso, independientemente del medicamento que esté recibiendo, por lo que podría aprovecharse como método de apoyo al manejo de base de este tipo de pacientes.

\section{Referencias}

1. Kaplan N . Hipertensión clínica. Buenos Aires: Médica Hispanoamericana, 1991;vol.1:1-40.

2.The Fifth Report of the Joint N ational Committe on Detection, Evaluation, and Treatment of High Blood Pressure. Arch Intern Med 1993;153: 154-183.

3. Working Group on Management of Patients with Hypertension and High Blood Cholesterol. National Education Programs Working Group Report on the Management of Patients with $\mathrm{H}$ ypertension and $\mathrm{High}$ Blood Cholesterol.Ann Intern Med 1991;114:224-237.
4. The Trials of Hypertension Prevention Collaborative Research Group. The effects of nonpharmacologic interventions on blood pressure of persons with high normal levels. Results of the trials of hypertension prevention, phase I. JAMA 1992;267:1213-1220.

5. 0 berman A, W assertheil-Smoller S, Langford HG, Blaufox D, Davis B, Blaszkowski $T$ et al. Pharmacologic and nutritional treatment of mild hypertension: Changes in cardiovascular risk status. Ann Intern Med 1990;112:89-95.

6. The Treatment of Mild Hypertension Research Group. The treatment of mild hypertension study. A randomized, placebo-controlled trial of a nutritional-hygienic regimen along with various drug monotherapies. A rch Intern Med 199|;151:1413-1423.

7. Kostis JB, Rosen RC, Brondolo E, Taska L, Smith D, W ilson A. Superiority of nonpharmacologic therapy compared to propanolol and placebo in men with mild hypertension:A randomized, prospective trial. Am Heart J 1992;123:466-474.

8.Applegate W B, Miller ST, Elam JT, C ushman W C, Derwi D, Brewer A et al. Nonpharmacologic intervention to reduce blood pressure in older patients with mild hypertension. Arch Intern Med 1992;152:1162-1166.

9. Croog SH, Levine S, Testa MA, Brown B, Bulpitt CJ, Jenkins D et al. The effect of antihypertensive therapy on the quality of life. $N$ Engl J Med 1986;314:1657-1664.

10. 0 rganización Panamericana de la Salud. La hipertensión arterial como problema de salud comunitario. W ashington, D.C.: O PS-O MS, 1990.

11. Fletcher $A E$, Hunt BM, Bulpitt $C J$. Evaluation of quality of life in clinic trials of cardiovascular disease. J Chron D is 1987:40:557-566.

12.A berg $\mathrm{H}$, Tibblin G .Addition of non-pharmacological methods of treatment in patients on antihypertensive drugs: Results of previous medication, laboratory test and life quality. J Intern Med 1989;226:39-46.

13. W assertheil-Smoller S. Biostatistics and epidemiology. A primer for health professionals. N ueva York: Springer-Verlag, 1991:86-103.

14. Morehouse LE, Miller A. Fisiología del ejercicio. 9a. edición. Buenos Aires: El Ateneo, 1976:255-261.

15. G uzmán J, Ponce de León S, Pita-Ramírez L, C astillo-Rentería C, PérezPimentel L. El cambio en la calidad de vida como indicador de curso clínico de la enfermedad. Comparación de dos índices. Rev Invest Clin 1993; 45:439-452

16. W assertheil-Smoller S, Blaufox D, $O$ berman A, D avis BR, Swencionis $\mathrm{Ch}, \mathrm{O}^{\prime} \mathrm{C}$ onnell $\mathrm{KM}$ et al. Effect of antihypertensives on sexual function and quality of life:The TAIM Study. Ann Intern Med 1991;114:613-620.

17. Cardiel MH. ¿Cómo se evalúa la calidad de vida? En: Moreno-Altamirano L, Cano-Valle F, García-Romero H, ed. Epidemiología clínica. 2a edición. México, D.F.: Interamericana-Mc Graw-Hill, 1994.

18. Grimm RH, Flack JM, Grandits GA, Elmer PJ, N eaton JD, Cutler JA et al. Long-term effects on plasma lipids of diet and drugs to treat hypertension. JAMA 1996;275:1549-1556.

19. N eaton JD, Grimm RH, Prineas RJ, Stamler J, Grandits GA, Elmer PJ et al.Treatment of mild hypertension study: Final results. JAMA 1993;270:713724.

20. Torres J. Educación participativa de la comunidad en cuidados perinatales. Aspectos metodológicos. Bol 0 ficina Sanit Panam 1987;102: 168-173. 